\title{
Angular spectral response from covered asphalt
}

\author{
Johan Casselgren,, ${ }^{1, \star}$ Mikael Sjödahl, ${ }^{1}$ and James LeBlanc ${ }^{2}$ \\ 1Division of Experimental Mechanics, Luleå University of Technology, 97187 Luleå, Sweden \\ 2Division of Signal Processing, Luleå University of Technology, 97187 Luleå, Sweden \\ ${ }^{*}$ Corresponding author: johan.casselgren@|tu.se
}

Received 13 November 2006; revised 21 February 2007; accepted 1 March 2007; posted 2 March 2007 (Doc. ID 77028); published 20 June 2007

\begin{abstract}
By measuring the spectral reflection from the four different road conditions dry, wet, icy, and snowy asphalt, a method of classification for the different surfaces-using two and three wavelengths-is developed. The method is tested against measurements to ascertain the probability of wrong classification between the surfaces. From the angular spectral response, the fact that asphalt and snow are diffuse reflectors and water and ice are reflective are confirmed. (c) 2007 Optical Society of America

OCIS codes: $240.6700,280.0280,300.6340,070.6020,290.5880$.
\end{abstract}

\section{Introduction}

A correlation between roadway condition and accident rate shows that roads with a low friction number have the highest statistics for accidents [1]. With increasing traffic density, road safety is an important issue. New vehicles have technical systems to prevent accidents, such as antilock brake systems (ABS) and electronic traction control (ETC), which step in when the vehicle loses traction. These systems could work better if the road condition in front of the vehicle could be estimated and incorporated into the system so that, for example, power could be reduced before the wheels lose traction. Preview sensors for this purpose are under development, and there are several prototypes for noncontact sensors for recognition of road conditions available using different methods. The main approach is to exploit the changes in reflected light under changing road surface conditions. An example of such a prototype is a TV-camera system combined with image processing [2]. This uses the fact that light reflected from a wet road becomes plane polarized, which makes it detectable. This technique is yet only tested for dry and wet roads. Another system uses laser diodes and photodetectors combined with signal processing to exploit the fact

0003-6935/07/204277-12\$15.00/0

(C) 2007 Optical Society of America that water, ice, and snow absorb light differently in the near-infrared (NIR) region [3]. This approach is also adopted for our investigation. A third method uses a different technique where the albedo (the ratio of incoming and reflected light) is measured with two pyranometers. This ratio is modulated by changing road conditions, and makes it possible to predict the road condition [4]. The purpose of this paper is to make spectrally and angularly resolved scattering measurements of the four road conditions-dry, wet, icy, and snowy asphalt-and determine their usefulness for setting up a classification algorithm. The objective is to extract the most information about the layer with the fewest wavelengths as possible to make a correct classification of the surface. This will be dependent on the direction of illumination and measuring position. Section 2 describes the experimental setup and the procedure measurement. Then, Section 3 shows the measurement result. Sections 4 and 5 describe a two- and three-wavelength analysis of the data, respectively. The paper ends with a discussion and conclusions.

\section{Experimental Setup}

Figure 1 shows the experimental setup [Fig. 1(a)] consisting of a halogen light for illumination, mounted together with a lens with $+150 \mathrm{~mm}$ focal length [Fig. 1(b)] giving a $50 \mathrm{~mm}$ light spot on the asphalt [Fig. 1(c)] positioned in the center of the (polymethyl- 


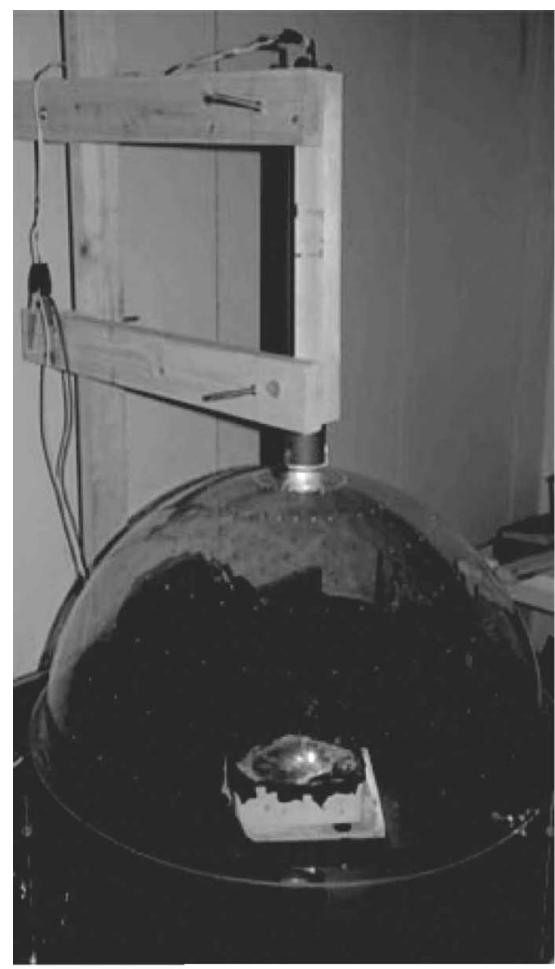

(a)

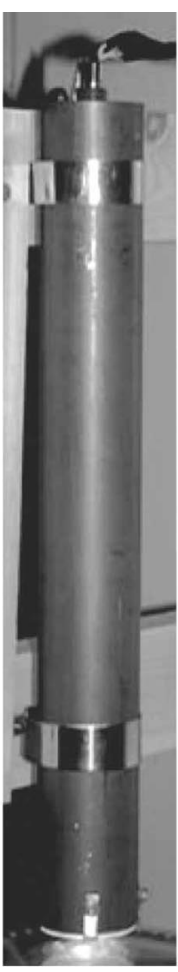

(b)

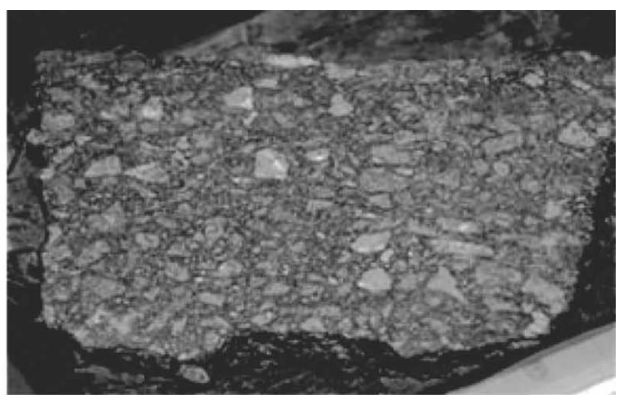

(c)

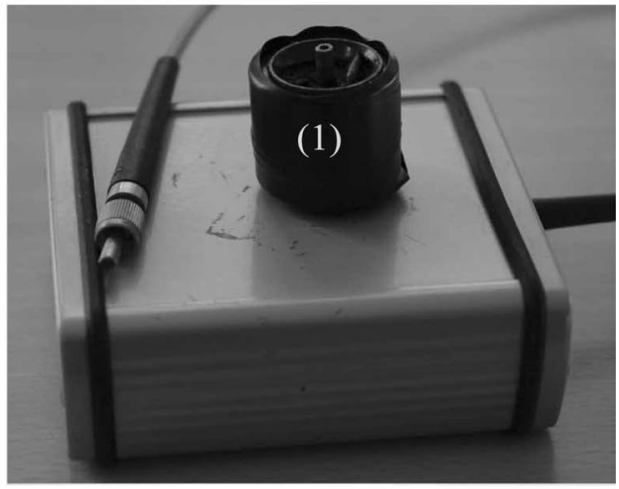

(d)

Fig. 1. (a) Half-sphere with the light source positioned at $0^{\circ}$. (b) Light source with the lens mounted at the end. (c) Piece of ABT 11 asphalt. (d) Spectrometer and the modifier numbered with (1).

methacrylate) half-sphere. The asphalt is a dense bitumen tar pulp (ABT 11) mixed with crushed stone components with the largest size of $11 \mathrm{~mm}$. The crushed stone changes depending on local assets of stone material, which also can alter the spectrum of the asphalt. We have chosen ABT 11 as it is common asphalt on Swedish roads representing $\sim 80 \%$ of all hot manufactured asphalt coatings in the country.

The half-sphere is drilled with holes with an angular resolution of $10^{\circ}$ from $0^{\circ}$ to $70^{\circ}$ vertically, where $0^{\circ}$ is perpendicular to the asphalt (see Fig. 2). Horizontally, it is drilled in entire circles around the sphere with an angular resolution of $10^{\circ}$ pointing at a spot at the center of the half-sphere. The holes are $3 \mathrm{~mm}$ in diameter to allow insertion of the

$(0,0)$

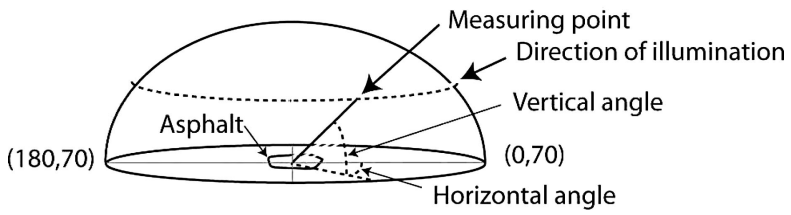

Fig. 2. Explanation as to how the measuring points and the directions of illumination are notated. The top point, perpendicular to the asphalt, is notated $(0,0)$ and the bottom $(0-180,70)$ because of the $70^{\circ}$ shift. Then, the vertical angle is shifted from $0^{\circ}$ to $180^{\circ}$ so the point on the opposite side of the backscattered light $(0,0-70)$ is notated with $(180,0-70)$. modified spectrometer [Fig. 1(d)] so it is perpendicular to the sphere's surface and measures on the same point at the center of the sphere. The spectrometer is a NIR 1.7 Spectrometer, from Boehiringer Ingelheim microParts $\mathrm{GmbH}$, which measures in the range of $1100-1700 \mathrm{~nm}$, with a numerical aperture of 0.22 . The spectrometer is modified with an extension [(1) in Fig. 1(d)] with a numerical aperture of 0.04 to have a field of view of $20 \mathrm{~mm}$ on the asphalt. Note that the measured spot is smaller than the light spot to ensure that the spectrometer measurements are restricted to within the illumination spot.

The spectrometer is set to measure the percentage of the reference light that is reflected from the surface. The measured intensities are the 16 bit digitized readout values of each pixel of the detector array. The measured value depends on the time of the integration and the intensity of the corresponding wavelength. The integration time is set to the maximum value to detect the reflected light from the water and ice outside the direct reflex. The spectrometer is corrected for the static mean value determined by the dark current offset of the diode array. The reference light is measured straight on the illumination at a distance twice the radius of the sphere. To get a good assessment of the layer's reflection, it is important that the experiments are carried out in the same way for all measurement cases. For that reason, a lift is placed under the asphalt to enable the top surface to always be at a 
radius distant from the sphere. Four different conditions are investigated; dry asphalt and asphalt covered with water, ice, and snow. The water used for both the water and ice layers is drinking water from the tap and should not contain any impurities. Altering the water depth was achieved by filling or emptying the container holding the asphalt sample. The level of water is measured at the same point on the asphalt, which is the zero level reference point for the rough surface of the asphalt. The water and snow depths are measured with a slide calliper. The same point is also used for the ice as zero level reference point, the ice thickness is measured with a micrometer.

The snow grains are filtered through two filters with hole sizes between 1 and $2 \mathrm{~mm}$, the distribution of the grains is assumed to be within this range. Since the snow is collected outdoors, it may contain some impurities, but it is assumed to represent pure snow. The grain shape is spherical. A sieve is used to apply the snow on the asphalt, resulting in a rough snow surface. In this case, an assessment is made of the snow depth. To preserve the ice and snow, the measurements are performed in a climate room with temperature $-10{ }^{\circ} \mathrm{C}$. Due to the cold for the personnel in the climate room, the measurements are limited to a $30^{\circ}$ angular resolution in the horizontal plane. This gives 7 measurements for every circle on the sphere, instead of 19 for the surfaces dry and water. The water and ice are measured for five different layers, moist or ice crystals, and a thickness of $1-4 \mathrm{~mm}$, the snow is only measured with thicknesses of 1 and
$3 \mathrm{~mm}$ because of problems in obtaining an even surface. The $3 \mathrm{~mm}$ snow surface is then pressed with an ice hockey puck and a weight of $3.4 \mathrm{~kg}$, to see if the spectrum changes between loose and dense snow. To minimize the measurements, the reflections are assumed to be symmetric so the measurements are constrained from $0^{\circ}$ (backscatter reflection) to $180^{\circ}$ (direct reflection). The direction of illumination is varied between $0^{\circ}$ and $70^{\circ}$ vertically. In the case where the direction of illumination and measuring point are the same, the measurement is done with a small change of the horizontal angle of $2^{\circ}-3^{\circ}$, due to the width of the light source. The results are analyzed in MATLAB.

\section{Measurement Results}

The four surfaces-dry asphalt and asphalt covered with water, ice, and snow-can be divided into fourteen layers; one for the dry, five for water and ice (moist and/or ice crystals, 1, 2, 3, and $4 \mathrm{~mm}$ ) and three for the snow (1, 3, and $3 \mathrm{~mm}$ compressed). Each layer is represented by 344 spectra of the reflected intensity in percentage of the reference intensity from the source of illumination. The 344 spectra are the result of measurements done with a $30^{\circ}$ angular resolution horizontally and $10^{\circ}$ vertically, which results in 43 measurements for each of the eight directions of illumination. Due to saturation of the spectrometer in the direct reflection for water and ice, discussed later in the paper, the horizontal angle $180^{\circ}$ is excluded from the averaging. To be able to compare the spectra, the dry and water measure-

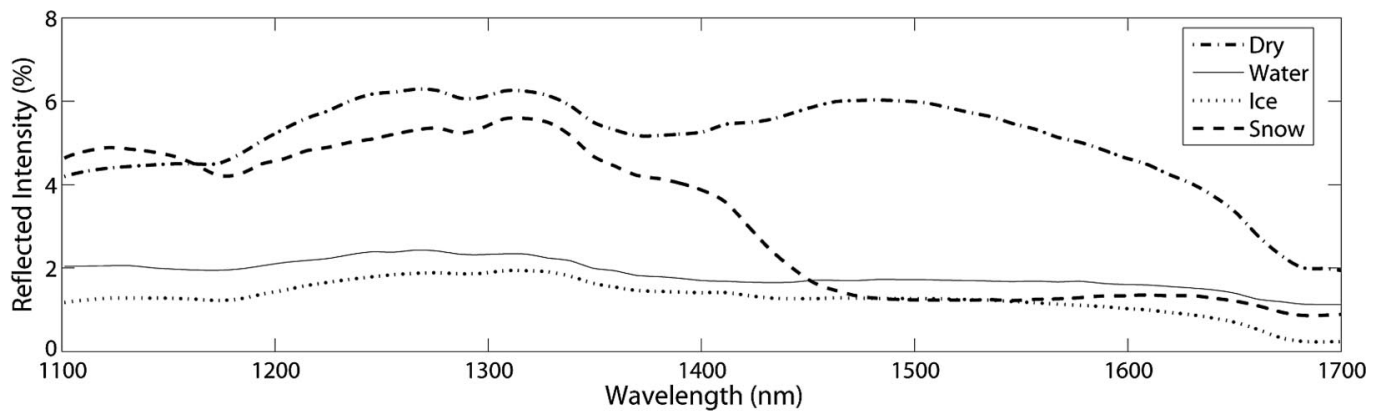

(a)

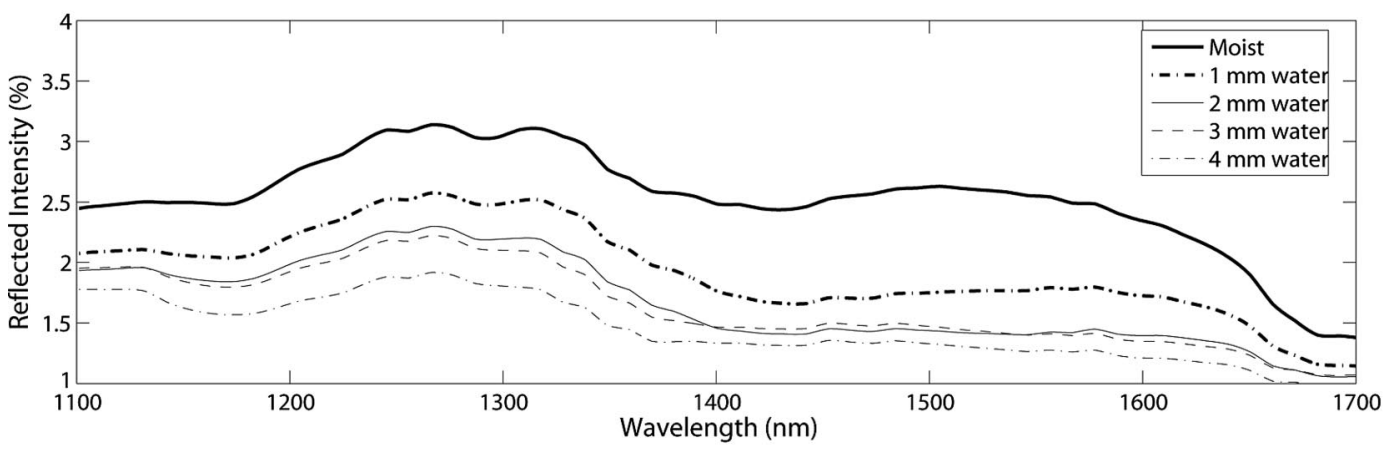

(b)

Fig. 3. (a) Characteristic spectrum for the surfaces dry, water, ice, and snow from the averaging over the 344 measurements for each layer. (b) Mean spectrum for the five different water depths. 
ments were reduced to an angular resolution of $30^{\circ}$ as the ice and snow. By averaging the 344 spectra for each layer, 14 spectra representing each layer are computed. Each surface is represented by a certain number of layers; by averaging the spectra of these layers, a characteristic spectrum is estimated for the four surfaces.

Figure 3(a) shows the characteristic spectrum of the surfaces, the characteristic reflection is due to the composition of the material. In Fig. 3(a), there are two different chemical components; the water molecule represented of water, ice, and snow but in different phases and the asphalt (which is composed of different components). The two media share some resemblance as they both consists of polar molecules, which are affected by light electromagnetic waves [5]. As an example, Fig. 3(b) shows the averaged spectrum for the layers of asphalt covered with various depths of water; all spectra were averaged for each wavelength. The plot shows that the reflected intensity level decreases as the water depth increases, which implies that detection of changing water depth on the asphalt is possible using the right wavelengths
[3]. Other measurements done on dry asphalt with other compositions of asphalts give a different spectrum [6] than the one shown in Fig. 3(a), which may be caused by a local variation in the composition of both the bitumen tar pulp and the crushed stone material. Measurements done on a random piece of asphalt result in a spectrum with the same characteristics, but with a different intensity level, as for the known asphalt which indicates that the spectral signature of the asphalt is correct.

A property of the different surfaces is the light scattering that is dependent on the top coating roughness and the composition of the medium. To analyze the difference between the four surfaces-dry, water, ice, and snow-the reflection intensity is compared. The measurements are taken at the wavelength 1310 $\mathrm{nm}$ and the direction of illumination $\left(40^{\circ}\right.$ from zenith) which is marked with an $\mathbf{x}$ in Fig. 4. The chosen wavelength represents one of the optimal wavelengths (to be discussed in Section 4) and the chosen direction of illumination shows the reflection characteristics well. The measurements show that there are no significant changes in the surface reflections

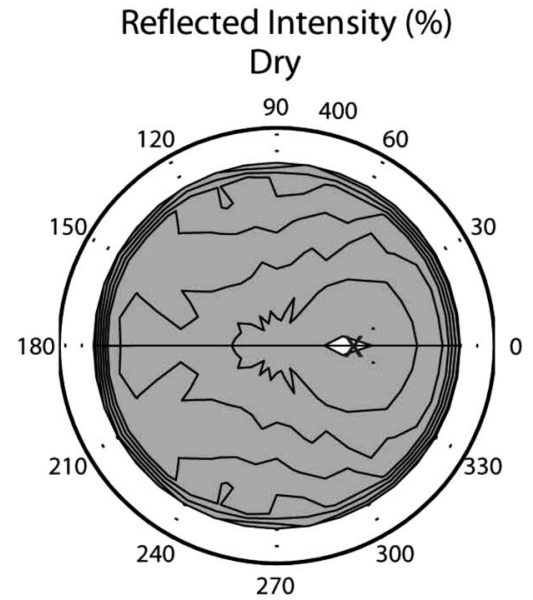

(a)

\section{Reflected Intensity (\%) Ice $3 \mathrm{~mm}$}

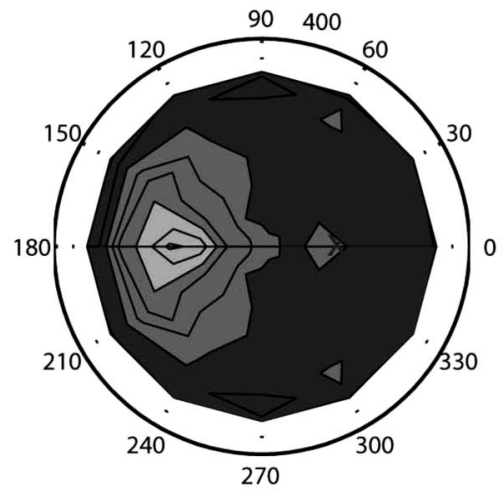

(c)

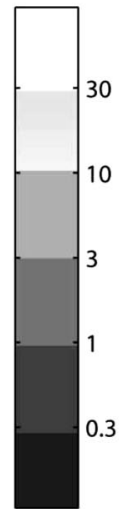

0.3

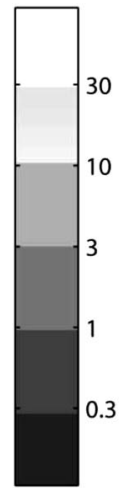

0.3

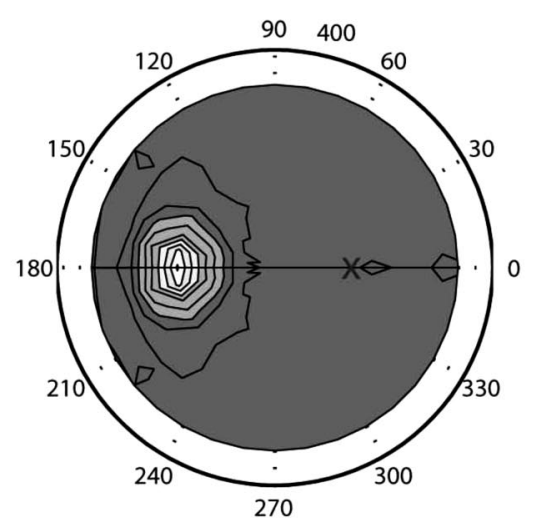

(b)

\section{Reflected Intensity (\%) Snow $3 \mathrm{~mm}$}
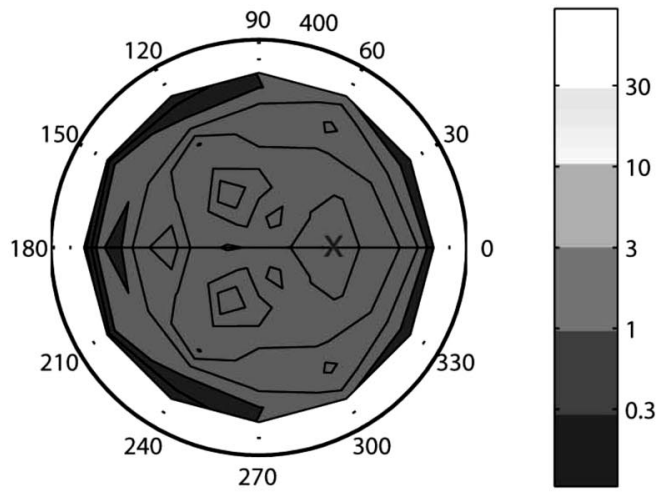

(d)

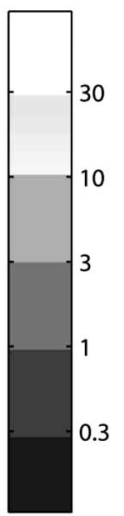

0.3

\section{3}

.

Fig. 4. Two-dimensional projection of the three-dimensional intensity response for the wavelength $1310 \mathrm{~nm}$ and the light position $40^{\circ}$ from the layers; (a) dry, (b) $3 \mathrm{~mm}$ water, (c) $3 \mathrm{~mm}$ ice, and (d) $3 \mathrm{~mm}$ snow. X marks the light position. 
due to different directions of illumination. To get the characteristics of the different surfaces without the affects of asphalt, the thickest snow layer $3 \mathrm{~mm}$ is chosen and the corresponding water and ice depths. The two-dimensional reflected intensity projections for the four layers are shown in Fig. 4. Note that the images are mirrored over the axis between $0^{\circ}$ and $180^{\circ}$. The figures describe the scattering of light in different horizontal and vertical angles. The horizontal angles are marked from $0^{\circ}$ to $360^{\circ}$, and the centers of the circles represent the vertical $0^{\circ}$ angle and the circumference represents $70^{\circ}$. As seen in Fig. 4, each layer has a characteristic scattering image depending on how the surface is composed. For dry asphalt and snow, the top coating is rough, which results in a diffuse scattering. The intensity level is almost the same over the whole area except at the edges where it decreases because of the direction of illumination. When the direction of illumination increases, the drop in intensity at the edges decreases. For the water and ice surfaces, the scattering is directed. A clear spot of high intensity is located at the opposite side from the direction of illumination, implying a reflective coating which smooths out the rough surface of the asphalt and thereby gives a distinct reflection peak. This reflection peak made the spectrometer to saturate because of the high intensity. The scattering image for water and ice are almost identical except from the reflected intensity level that is lower for ice than for the water, which indicates that ice absorbs more of the electromagnetic waves at that wavelength, this is also seen in Fig. 3.

\section{Two-Wavelength Data Analysis}

In a realized sensor for automotive applications, it is of interest to keep the number of wavelengths to analyze as low as possible. The calculations for such a sensor have to be at a minimum to achieve acceptable response times. Otherwise, the sensor can not measure the road surface ahead of the vehicle, which is the point of a preview sensor. The sensor also needs to be robust for intensity changes that can be caused by a number of factors (changes of distance to the measuring point, a bump, or changes in the reflection as for new and old asphalt or dirty sensors). One solution to this problem is to assume that the intensity change is the same over all wavelengths. The ratio of two wavelengths is thus insensitive to intensity changes. However, when the road surface changes, the ratio will indeed be altered. It is therefore of interest to determine two wavelengths whose ratio has the largest variance for the four different road conditions-dry, water, ice, and snow. Therefore, we choose to represent the measurements in matrices for each surface according to Eq. (1), where the ratios between all wavelengths are represented. For this computation, the averaged spectra for each surface from Fig. 3(a) was used because they represent all measurements over the angular distribution. To calculate the variance between the ratios for the four surfaces, first the mean value was calculated for each element according to Eq. (2) and then the variance $V$ in Eq. (3).

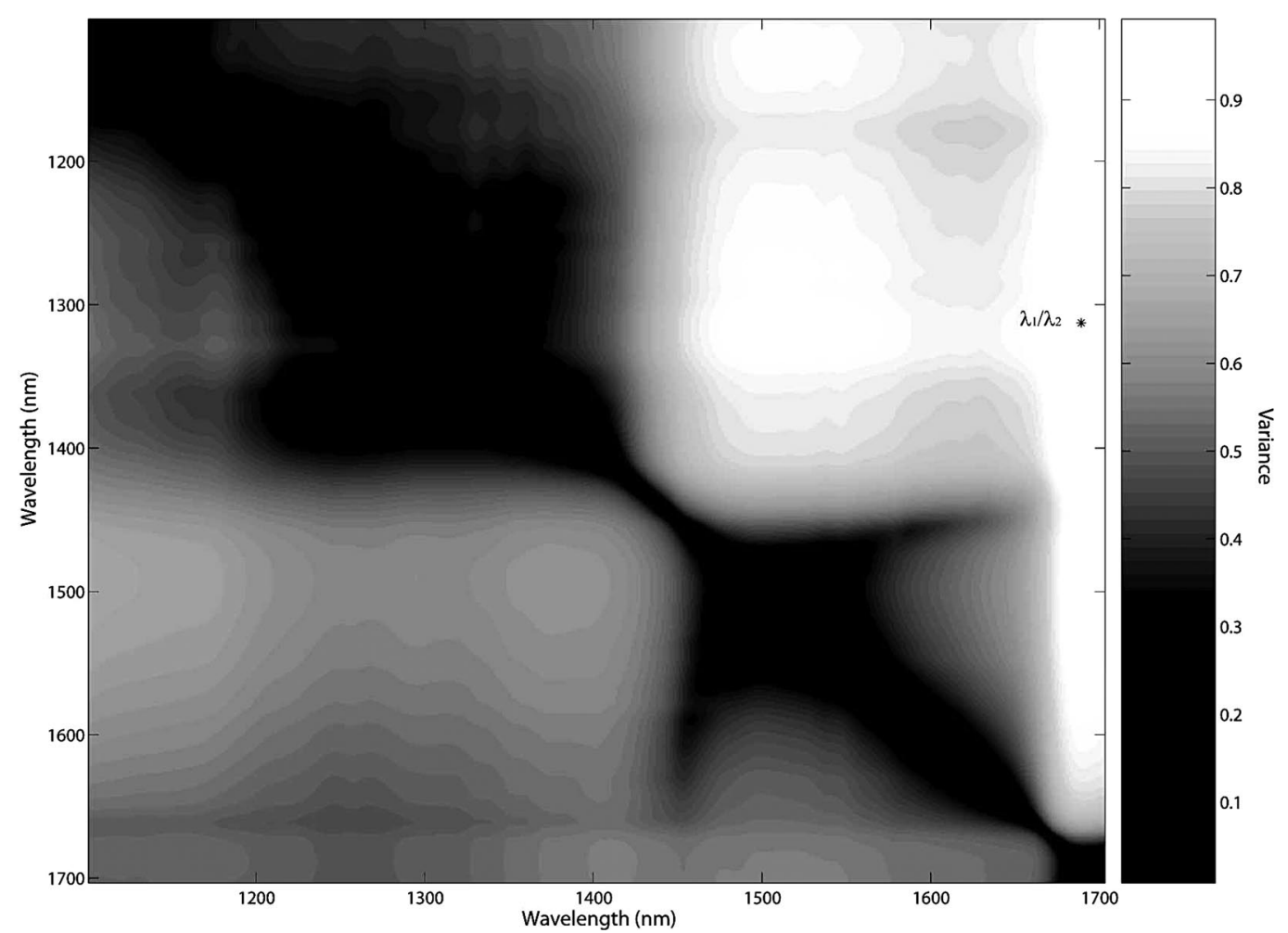

Fig. 5. Variance of the wavelength ratio for the four surfaces, with the optimal wavelengths marked with *. 


$$
\begin{gathered}
X_{\text {Dry }}=\left[\begin{array}{ccc}
\frac{\lambda_{1100}}{\lambda_{1100}} & \cdots & \frac{\lambda_{1100}}{\lambda_{1700}} \\
\vdots & \ddots & \vdots \\
\frac{\lambda_{1700}}{\lambda_{1100}} & \cdots & \frac{\lambda_{1700}}{\lambda_{1700}}
\end{array}\right], \quad X_{\text {Water }}=\left[\begin{array}{ccc}
\lambda_{1100} & \cdots & \lambda_{1100} \\
\frac{\lambda_{1100}}{\lambda_{1700}} & \ddots & \vdots \\
\vdots & \ddots & \lambda_{1700} \\
\frac{\lambda_{1700}}{\lambda_{1100}} & \cdots & \frac{\lambda_{1700}}{\lambda_{1700}}
\end{array}\right], \\
X_{\text {Ice }}=\left[\begin{array}{ccc}
\lambda_{1100} & \cdots & \lambda_{1100} \\
\frac{\lambda_{1100}}{\lambda_{1700}} \\
\vdots & \ddots & \vdots \\
\frac{\lambda_{1700}}{\lambda_{1100}} & \cdots & \frac{\lambda_{1700}}{\lambda_{1700}}
\end{array}\right], \quad X_{\text {Snow }}=\left[\begin{array}{ccc}
\frac{\lambda_{1100}}{\lambda_{1100}} & \cdots & \frac{\lambda_{1100}}{\lambda_{1700}} \\
\vdots & \ddots & \vdots \\
\frac{\lambda_{1700}}{\lambda_{1100}} & \cdots & \frac{\lambda_{1700}}{\lambda_{1700}}
\end{array}\right],
\end{gathered}
$$

$$
\begin{aligned}
\bar{x}[i, j]= & \frac{1}{4}\left(X_{\text {Dry }}[i, j]+X_{\text {Water }[}[i, j]+X_{\text {Ice }}[i, j]\right. \\
& \left.+X_{\text {Snow }}[i, j]\right),
\end{aligned}
$$

for $i=1,2,3, \ldots, 581$ and $j=1,2,3, \ldots, 581$.

The spectral resolution of the spectrometer is 581 between 1100 and $1700 \mathrm{~nm}$; for that reason, $i$ and $j$ are from 1 to 581 .

$$
\begin{aligned}
V[i, j]= & \frac{1}{3}\left(\left(X_{\text {Dry }}[i, j]-\bar{x}[i, j]\right)^{2}+\left(X_{\text {Water }}[i, j]-\bar{x}[i, j]\right)^{2}\right. \\
& \left.+\left(X_{\text {Ice }}[i, j]-\bar{x}[i, j]\right)^{2}+\left(X_{\text {Snow }}[i, j]-\bar{x}[i, j]\right)^{2}\right),
\end{aligned}
$$$$
\text { for } i=1,2,3, \ldots, 581 \text { and } j=1,2,3, \ldots, 581 \text {. }
$$

The variable $V$ [Eq. (3)] is then scaled and imaged in Fig. 5. Finding the maximal variance between the four surface's wavelength ratio gives two optimal wavelengths for classification. The calculations shows that the maximum variance is at $\lambda_{1}=1310 \mathrm{~nm}$ and $\lambda_{2}$ $=1690 \mathrm{~nm}$, which is set to the optimal wavelengths. Also notable is that the diagonal in Fig. 5 is zero because of the diagonals in the matrixes $X_{\text {Dry }}, X_{\text {Water }}$, $X_{\text {Ice, }}$, and $X_{\text {Snow }}$ all are one. As seen in Fig. 5, except the large maximum point for the two optimal wavelengths, there is one smaller maximum value at 1310 and $1490 \mathrm{~nm}$, where $1310 \mathrm{~nm}$ is one of the optimal wavelengths. These two wavelengths are the common commercial wavelengths for lasers used in fiber optics; therefore, they are mass produced and easy and cheap to buy. In a commercial sensor, this is of interest where the cost needs to be at a minimum.

An important issue for a preview sensor is at what angle it should be mounted to work as fast and reliably as possible. The angle should be flat to detect the surface ahead of the vehicle as faraway as possible to achieve the longest prediction time. But this can be conflicted with the best angle for surface classification. By computing and finding the maximum value of the variance as in Eqs. (1)-(3), but only for the spectra represented by a certain measuring point and direction of illumination, we are able to compare the different light and measuring positions and find the best angles for classification. The result of the calculations is shown in Fig. 6, where each line represents

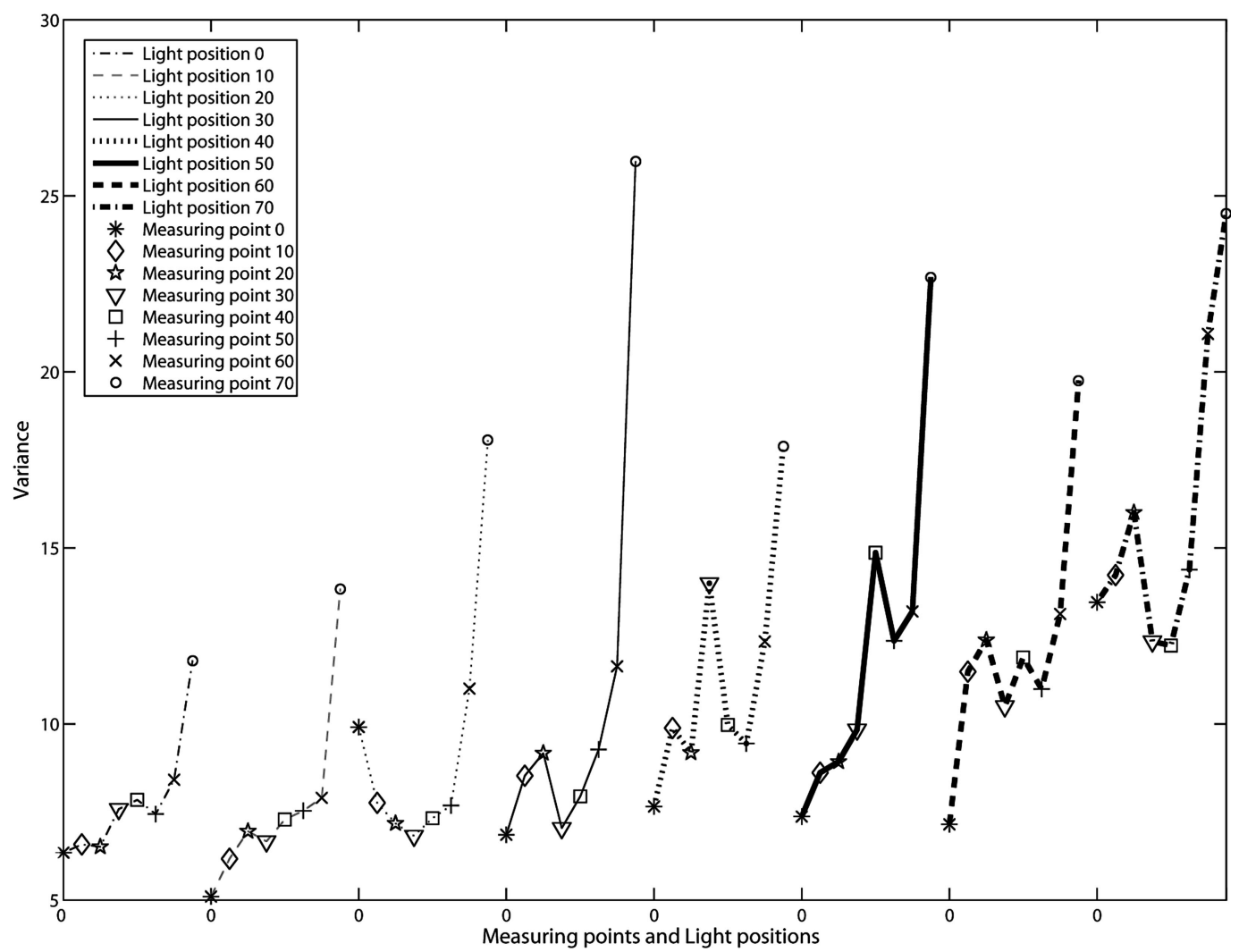

Fig. 6. Maximum points of the wavelengths ratio variance between the four surfaces (the markers) for the eight directions of illumination of the backscattered light; each curve represents a light position. 
Reflected Intensity (\%) Snow $3 \mathrm{~mm}$ wavelength $1310 \mathrm{~nm}$

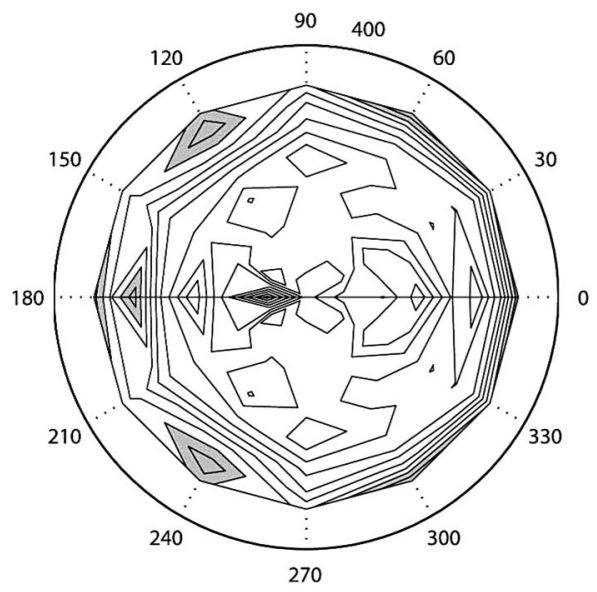

(a)
Reflected Intensity (\%)

Snow $3 \mathrm{~mm}$ wavelength $1690 \mathrm{~nm}$

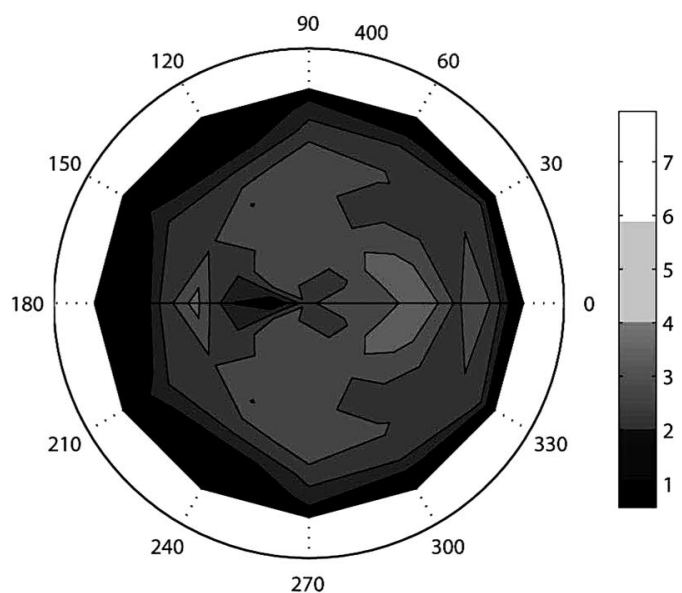

(b)

Fig. 7. Reflected intensity at the light position $40^{\circ}$ and for the two wavelengths $\lambda_{1}=1310 \mathrm{~nm}$ and $\lambda_{2}=1690 \mathrm{~nm}$ for the $3 \mathrm{~mm}$ layer of snow.

a direction of illumination and each marker represents a measuring point. The interpretation of Fig. 6 is that for all eight directions of illumination the mea- suring point $70^{\circ}$ gives the maximum value of the variance. The best light position is either $30^{\circ}$ or $70^{\circ}$ for these measurements. In this case, the $70^{\circ}$ is pref-

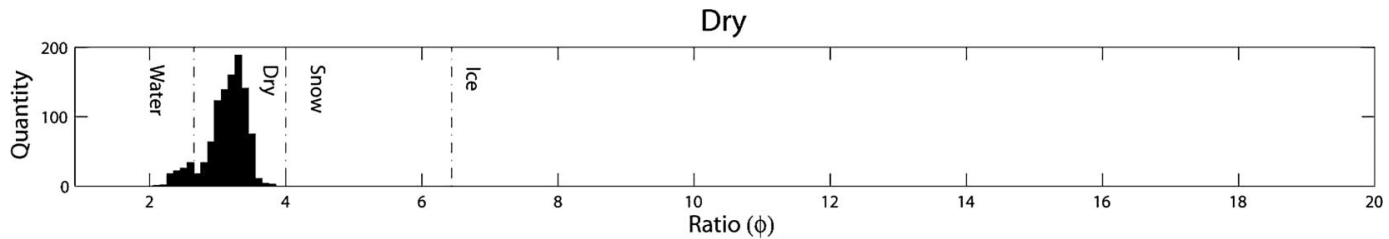

(a)

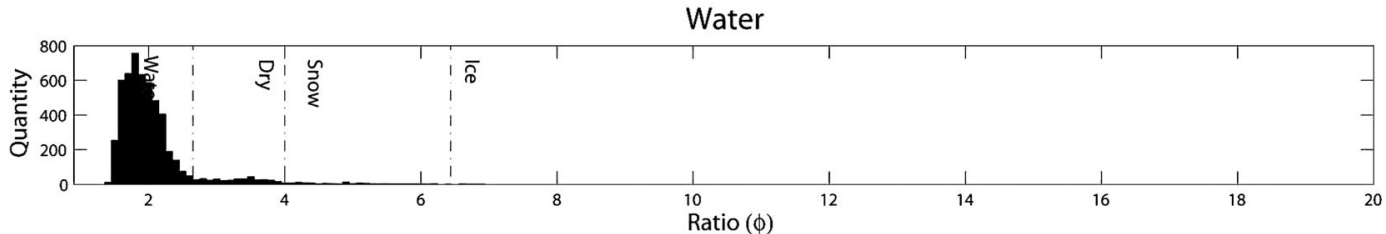

(b)

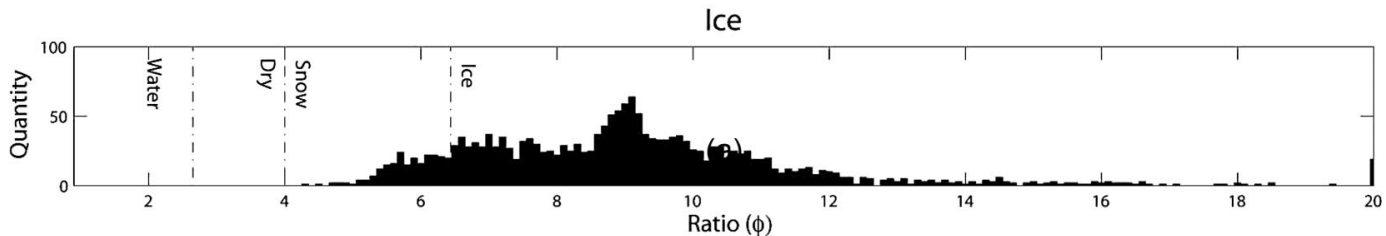

(c)

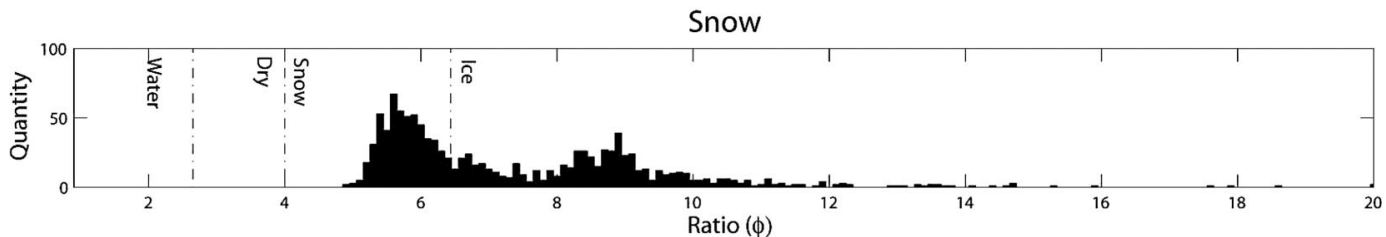

(d)

Fig. 8. Histogram for the scattering plot for the 9520 measuring points for the four different road conditions for the ratio $(\phi)$. 
erable because it results in a longer prediction time. Also notable in Fig. 6 is that all curves have an increasing trend for the last measuring point of $70^{\circ}$. It is possible that a measuring point at $80^{\circ}$ might result in a higher variance for the direction of illumination $70^{\circ}$. The calculations also show that the maximum variance for each light and measuring point agreed with the optimal wavelengths 1310 and $1690 \mathrm{~nm}$ with a difference of $2-3 \mathrm{~nm}$. This shows that the averaged calculation of the optimal wavelengths is a good representation of the measurements.

An interesting effect of snow is that the reflected intensity scattering from the snow gets almost "black" above $1450 \mathrm{~nm}$ as seen for the optimal wavelengths in Fig. 7 (the figure is created in the same way as Fig. 4). Also note in Fig. 7 that the intensity for the wavelength $1690 \mathrm{~nm}$ decreases as the angle approaches $70^{\circ}$, unlike in the $1310 \mathrm{~nm}$ case. The result of the intensity change is an increase in the ratio between the two wavelengths as the angle increases. This explains why the variance between the ratios also increases in Fig. 6 with the angle, this result implies that for an automotive application the light source should be placed above (with a smaller angle) the detector to achieve a more reliable classification.
Also noted is that the dense snow shows the same spectral response as the loose snow. Thus, the corresponding scattering plot appears to be the same for both loose and dense snow.

To investigate the classification of the four different surface conditions by using two wavelengths the ratio $\phi=\lambda_{1} / \lambda_{2}$ is introduced and calculated for all measuring points. Figure 8 shows the resulting distributions for each surface using histograms. From the distributions, it is possible to set up classification boundaries $B$ for the four surfaces (shown as the vertical dotted lines). These boundaries are determined by finding the minimum point of the function $f$ in Eq. (4). As an example, the boundaries between dry and water [Figs. 8(a) and 8(b)] are found as

$$
\begin{aligned}
& f[B]=\sum_{n=\phi_{\min }}^{B} D_{A}{ }^{D}[n]+\sum_{n=B}^{\phi_{\max }} D_{A}{ }^{W}[n] \\
& \text { for } B=2,2.001,2.002, \ldots, 3, \\
& B_{\min }=\arg _{B} \min f[B],
\end{aligned}
$$

where $D_{A}{ }^{D}$ is the dry distribution and $D_{A}{ }^{W}$ is the water distribution and, for this case, $B$ is from 2 to 3 and $\phi_{\min }=0$ and $\phi_{\max }=20$, as seen in Figs. 8(a) and

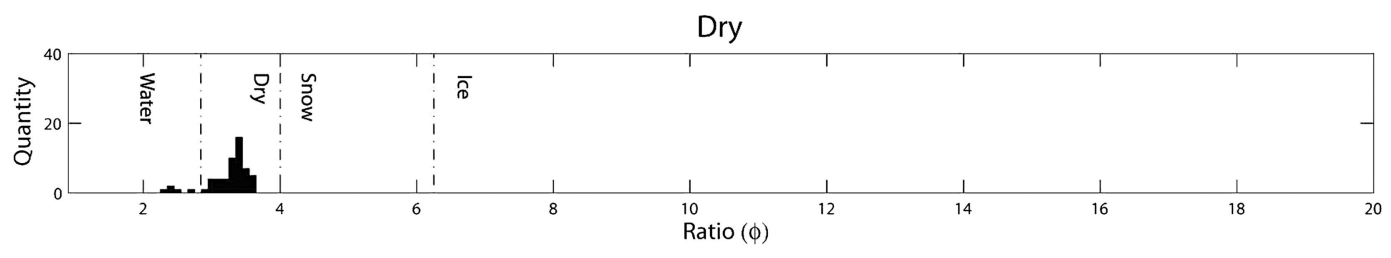

(a)

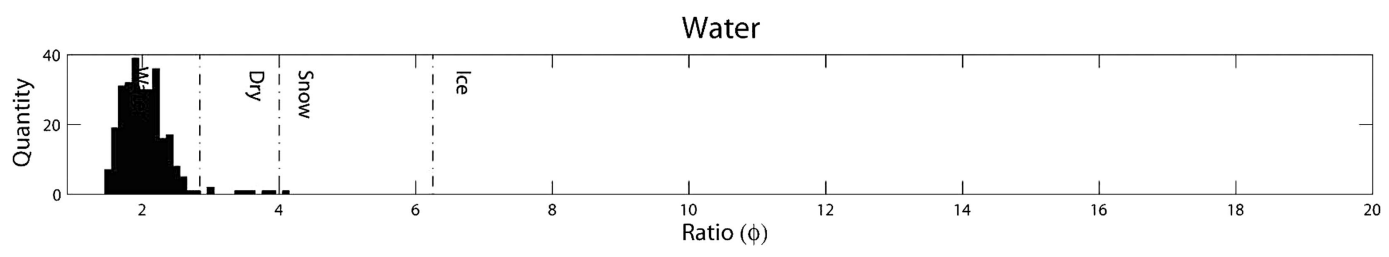

(b)

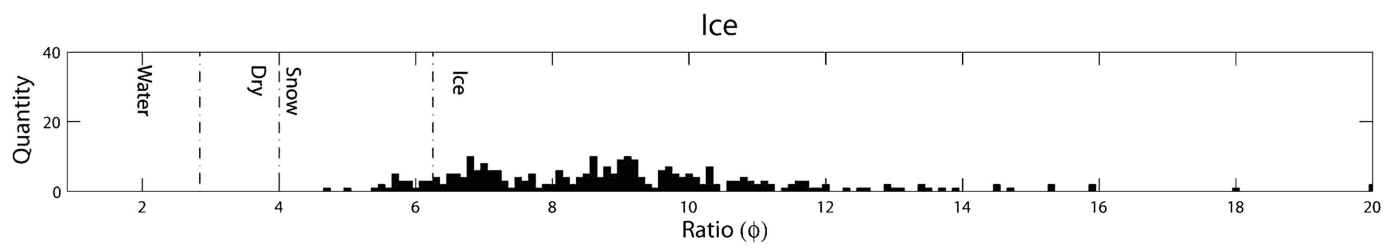

(c)

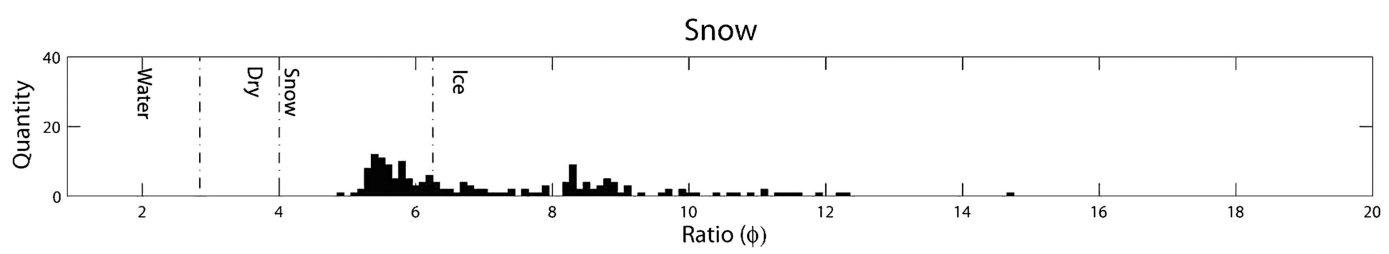

(d)

Fig. 9. Histogram for the scattering plot for 784 measuring points for the backscattered light for the four different road conditions the ratio $(\phi)$. 


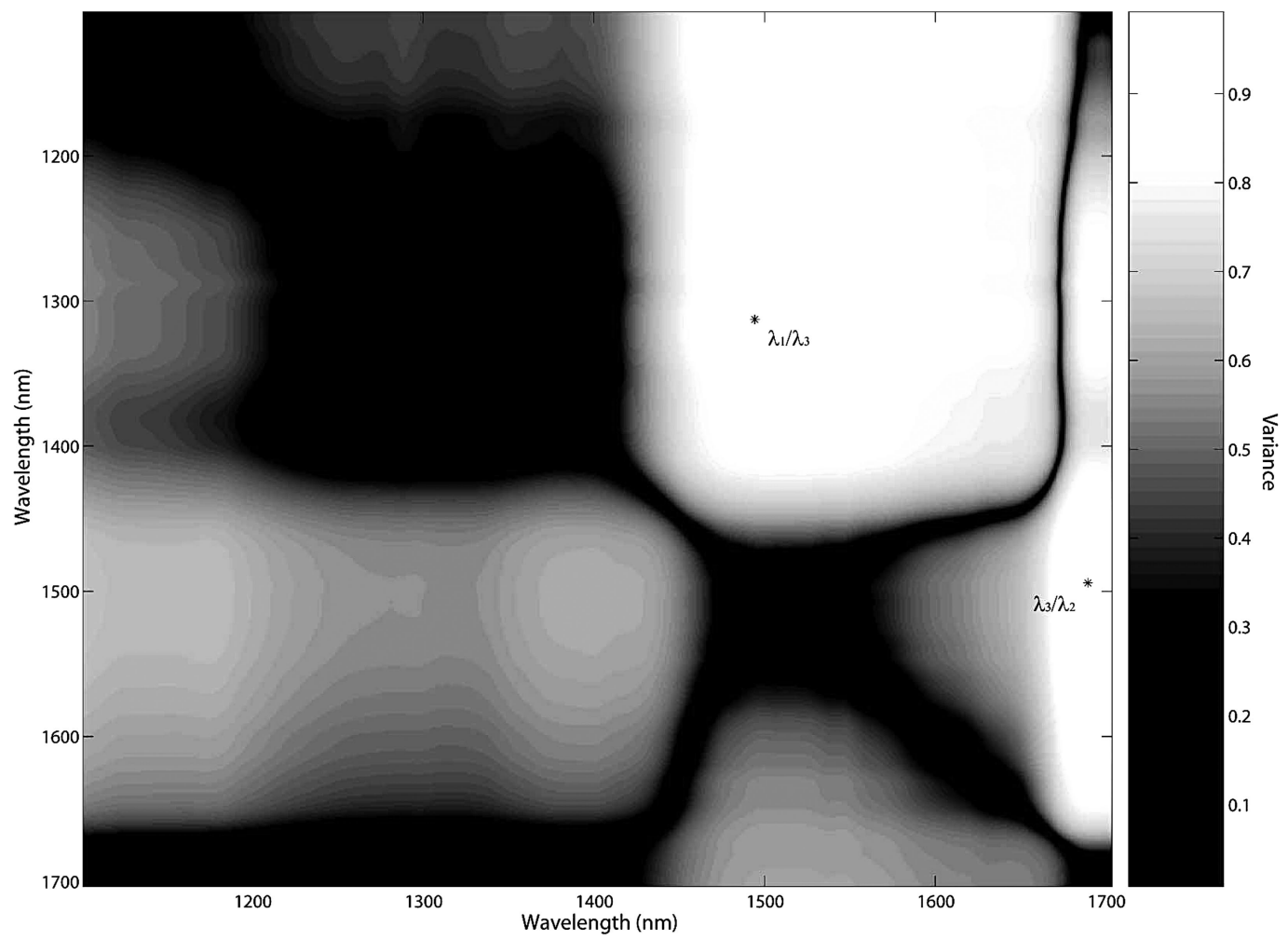

Fig. 10. Variance between mean ratios for the surfaces ice and snow.

8(b). The boundary is found by finding the minimum argument for $B$.

Figure 8 shows that the distributions for ice and snow overlap considerably, which makes classification (and proper boundary determination) difficult. Separating the dry asphalt and water covered surfaces are not as difficult, although there are some measurements of the water inside the dry boundaries. Such measurements lying outside the selected boundaries will imply the possibility of wrong classification $\left(P_{\text {wrong }}\right)$. In fact, the consequences of such erroneous classification should be taken into account when determining the classification boundaries in an application scenario. For a preview sensor to work accurately, $P_{\text {wrong }}$ should be low. To determine $P_{\text {wrong }}$ for the data measurements and the selected boundaries, we write

$$
\begin{aligned}
P_{\text {wrong }}= & P(\text { Ice } \mid \text { Snow })+P(\text { Snow } \mid \text { ice })+P(\text { Water } \mid \text { Ice }) \\
& +P(\text { Ice } \mid \text { Water })+P(\text { Water } \mid \text { Dry }) \\
& +P(\text { Dry } \mid \text { Water })+P(\text { Ice } \mid \text { Dry })
\end{aligned}
$$

where $P($ Ice $\mid$ Snow $)$ denotes the probability of classifying measurement as "Ice" when the correct surface is "Snow." This probability may be seen as a measure of the reliability of the classification method. For the case of all measurements with the selected boundaries as in Fig. 8, we have $P_{\text {wrong/all }}=15 \%$.

For a preview sensor, the idea is to survey the road ahead of the vehicle and to work as fast as possible, which implies that it should only use beam width of $0^{\circ}-5^{\circ}$ (backscattered light) to be able to measure in front of the tire. Another benefit is that the optical path for the light to propagate is minimized. It is possible to use horizontal angles larger than $0^{\circ}-5^{\circ}$, but this angle is then limited by the width of the vehicle and makes the response time longer. Figure 9 shows a histogram for the scattering plot of 784 measuring points. Using only the backscattered light, the number of measurements changes from 9520 to 784 ; because of this, the distribution's (shown in Fig. 8) variance and median change, which results in a change of boundaries. Using the boundaries for the backscattered light, the $P_{\text {wrong/back }}$ becomes $16 \%$, which is larger than in the case of all measurements.

\section{Three-Wavelengths Data Analysis}

The result of the two-dimensional data analysis showed that two wavelengths have a limited ability to accurately classify the road surfaces. To understand this limitation, we look at how the four surfaces are distributed within the boundaries in occurrences of wrong classification. Table 1 shows for which surfaces the two wavelengths are dominant, meaning that they have a low $P_{\text {wrong. }}$. It also shows that using $\lambda_{1}$ and $\lambda_{2}$ results in a high probability of wrong classification for snow. Figures 8(c) and 8(d) show that the separation of ice and snow with ratio $\phi$ is not sufficient because of the overlapping distributions. Therefore, an analysis of the ratio variance for only ice and snow is calculated to investigate the optimal wavelengths for separating the ice and snow distributions. The conclusion of Fig. 10 is that a third wavelength $\left(\lambda_{3}=1490 \mathrm{~nm}\right)$ needs to be added to the calculations. 
Table 1. Probability of Wrong Classification for All Surfaces and for Each Surface

\begin{tabular}{|c|c|c|c|}
\hline & $\begin{array}{l}P_{\text {wrong }} \\
\quad(\%)\end{array}$ & $\begin{array}{l}\text { Quantity of } \\
\text { Measurements } \\
\text { Outside the } \\
\text { Boundaries }\end{array}$ & $\begin{array}{c}\text { Quantity of } \\
\text { Measurements }\end{array}$ \\
\hline All surfaces & 14 & 1333 & 9520 \\
\hline Dry & 9 & 96 & 1064 \\
\hline Water & 9 & 479 & 5320 \\
\hline Ice & 11 & 216 & 1960 \\
\hline Snow & 54 & 635 & 1176 \\
\hline
\end{tabular}

As seen in Fig. 10, the ratios $\lambda_{1} / \lambda_{3}$ and $\lambda_{3} / \lambda_{2}$ result in maximum values, although $\lambda_{3} / \lambda_{2}$ is larger than $\lambda_{1} / \lambda_{3}$, but calculations show that a combination of the ratio between $\lambda_{1} / \lambda_{3}$ and $\phi$ results in the lowest $P_{\text {wrong }}$, therefore introducing the ratio $\theta=\lambda_{1} / \lambda_{3}$. Calculations also reveal that the best result is achieved by first using the $\theta$ to separate the dry and snow measurements from water and/or ice (see Fig. 11 left column), then separating water and ice with the $\phi$ (see Fig. 11 right column). The result of the two ratio calculations is shown by the histograms in Fig. 11, where the right column for the $\phi$ is the same as in Fig. 8 , except that first the $\theta$ is used to eliminate the measurements outside the boundaries for each surface, the outliers. The measurements outside the water and/or ice boundaries, in the left column for the $\theta$, are eliminated so they do not get registered twice as wrong classifications. As shown in the twowavelength data analysis, the ice and snow were difficult to separate; however, using two ratios and only three wavelengths, it is possible to separate them with a low $P_{\text {wrong }}$. In this case, $P_{\text {wrong/all }}$ drops from $14 \%$ to $4 \%$. Comparing $\theta$ and $\phi$ shows that no matter which of the two ratios are chosen, there are always two surface distributions that are overlapped [see Figs. 11(b), 11(c), 11(g), and 11(h)]; in the left column, it is water and ice and in the right it is ice and snow. The histograms in Fig. 12 show the result from the 784 backscatter measurements of interest for a realized preview sensor. As seen in Fig. 12, there are only a few measurements that are outside the boundaries-and as in the case of all measurements, these measurements for water and

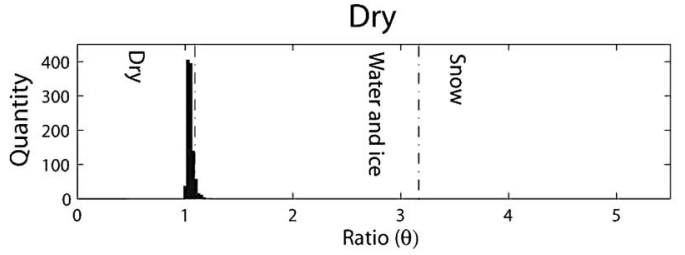

(a)

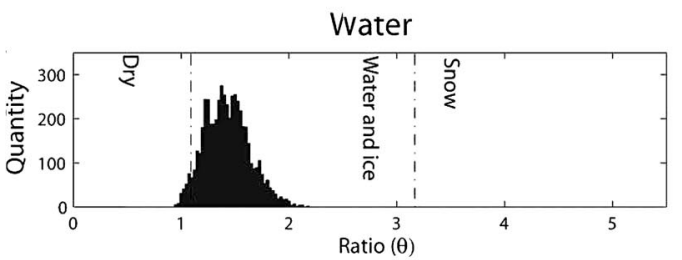

(b)

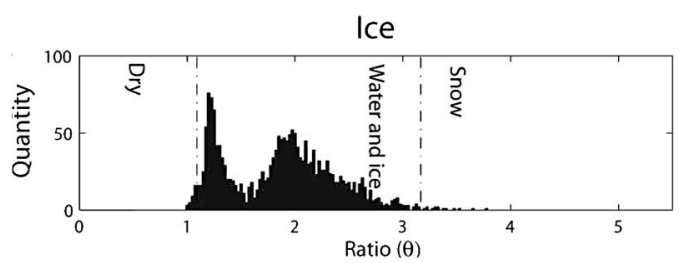

(c)

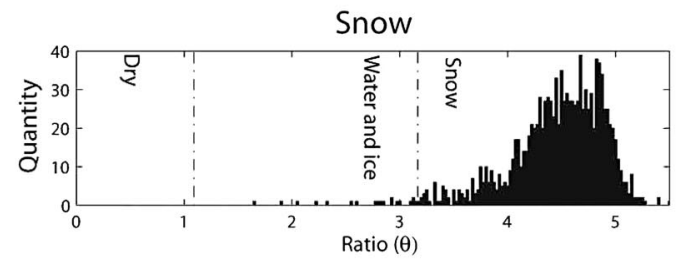

(d)

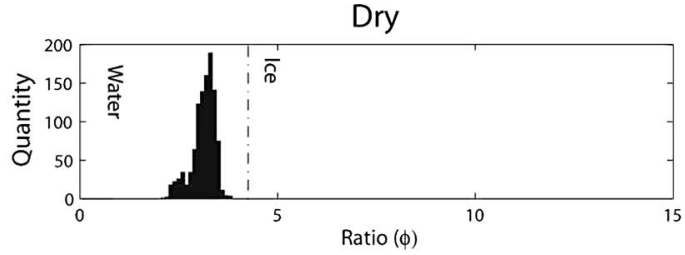

(e)

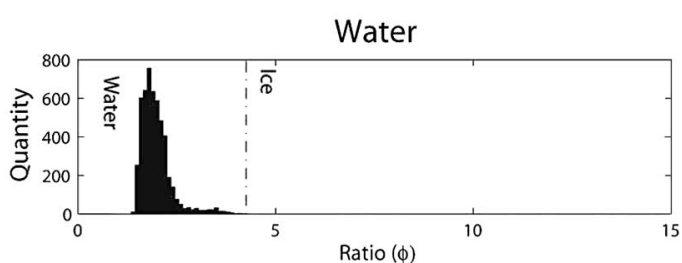

(f)

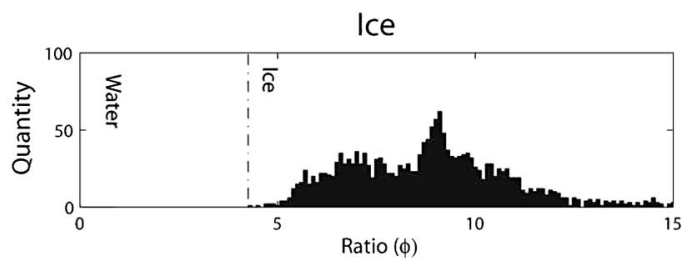

(g)

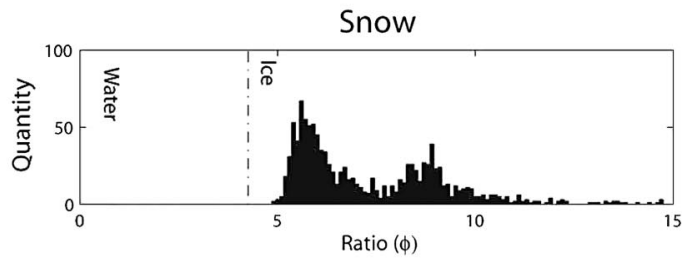

(h)

Fig. 11. Histogram for the scattering plot for 9520 measuring points for the four different road conditions. The left column: Ratio ( $\theta$ ). The right column: Ratio $(\phi)$. 


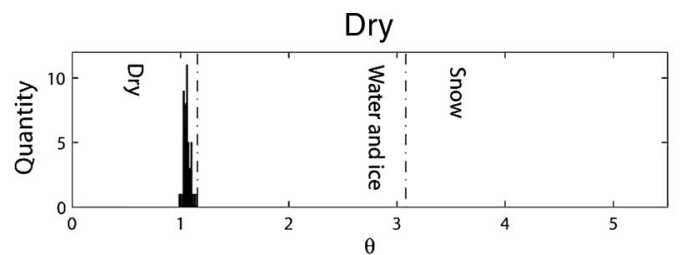

(a)

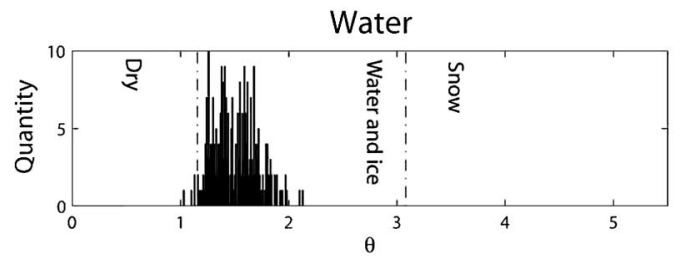

(b)

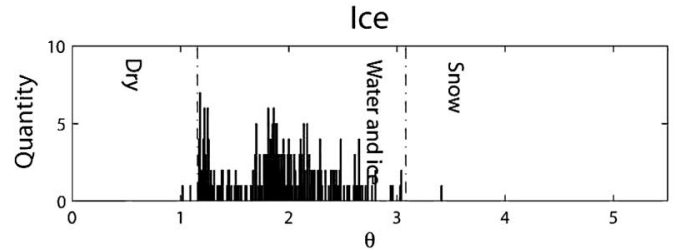

(c)

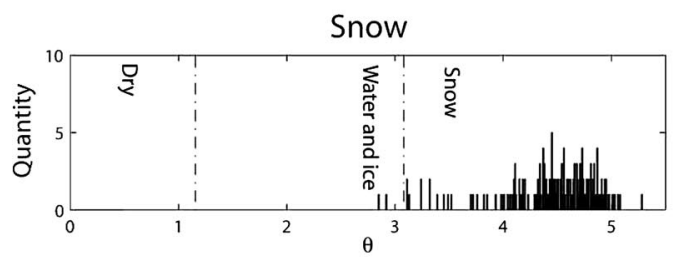

(d)

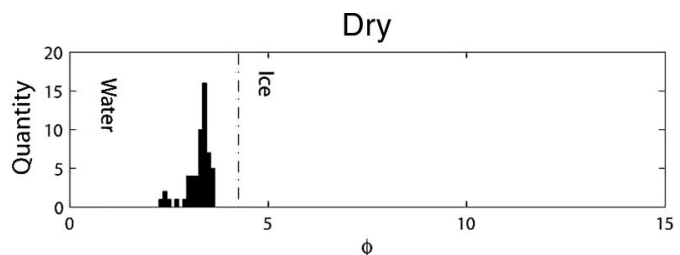

(e)

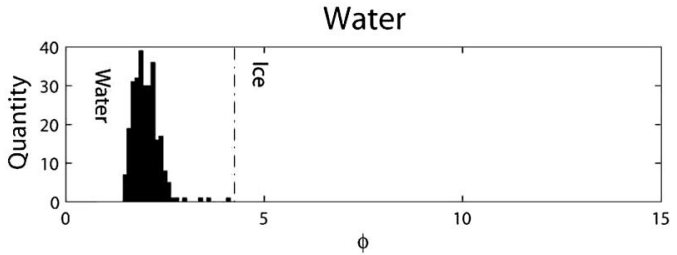

(f)

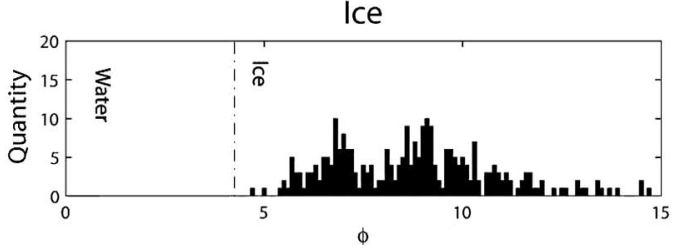

(g)

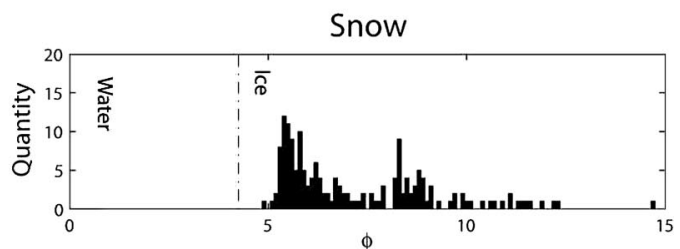

(h)

Fig. 12. Histogram for the scattering plot for 784 measuring points for the four different road conditions. The left column: Ratio $(\theta)$. The right column: Ratio $(\phi)$.

ice are eliminated and the residual measurements for water and ice are totally separable [Figs. 12(f) and $12(\mathrm{~g})]$. This results in a $P_{\text {wrong } / \text { back }}=1 \%$, which may be acceptable for applications. The summation of the three-wavelength analysis is that for a static case so that the classification yields satisfactory results.

\section{Discussion}

The averaging for the different spectra are done for the angular domain over the measuring points on the hemisphere. The unit of the averaged spectrum for each surface is an average estimation of the percentage of the reflected intensity of the reference intensity from the illumination source. Except for the direct reflection from the illumination source for the water and ice surfaces, the intensities are almost uniform for all measuring points as Fig. 4 shows for the four surfaces. This implies that an angular distribution average of the spectra is an acceptable estimation for each wavelength and surface.

The spectral plots in Fig. 3 show that the various surfaces have characteristic spectra, which make it possible to detect changes in road condition. Comparing plots of pure water and ice transmission found in literature is not the same as the spectrum of water and ice shown in Fig. 3. This implies that the spectra of water and ice are affected by the asphalt, because liquid water and ice are transparent media. First, at a depth of around $10 \mathrm{~mm}$ of water, the spectrum approaches the inverted plot of the absorption for water. A comparison between the measured spectrum for snow and the spectra for different sizes of pure snow crystals found in literature [7] gives the conclusion that the spectrum looks the same for pure snow as for snow on asphalt. This constitutes the well-known fact that snow is not a transparent medium and is not affected by the asphalt as water and ice. The compressing of the snow to analyze the difference between dense and loose snow shows that there is no significant difference between the two. In this case, the three layers of snow show no difference but if the pressure is higher, for example, such as from a truck, and some heat is added from the wheels the spectrum could change because the top surface gets a more reflective and smooth surface. This has not been investigated more closely herein.

The two-dimensional projections in Fig. 4 show that water-ice and dry-snow scatter light in two dif- 
ferent ways. Unfortunately the two surfaces-water and ice- that are hardest to separate have the same scattering characteristics. One thing that separates the two surfaces is the temperature, so one course of action could be combining a two- or three-wavelength sensor with a surface temperature measurement to decrease the wrong classifications. Figure 4 also shows that the direct reflection intensity is much higher for water and ice than for the other angles and surfaces, but the distribution for these measurements is the same as for the other angles. This is an example of the robustness of the ratio calculations because intensity change is the same for the whole spectra so the ratio stays the same.

Using the wavelength at $1690 \mathrm{~nm}$ could be objected to because of the vicinity of the end of the measuring band of the spectrometer. But the accessible range for the spectrometer is $1050-1750 \mathrm{~nm}$ so the wavelength is within the range. The drop in the transmitted intensity for the dry, water, and ice spectra at 1650-1700 nm shown in Figs. 3(a) and 3(b) (water and ice alone) can be attributed to first-order overtones and combinations of various $\mathrm{C}-\mathrm{H}$ stretching fundamentals [8] that appear distinctively for asphalts with high bitumen content. This also affects the transparent media water and ice, so this is not an effect of spectrometer. The fourth surface snow with a grain size of 1-2 $\mathrm{mm}$ also has a known drop $\sim 1650-1700 \mathrm{~nm}$ [7], shown in the spectra in Fig. 2 (a), this is also a verification of the spectrometer. This supports the use of the third wavelength at $1690 \mathrm{~nm}$.

The result of the analysis of the measurements shows that an implementation in a sensor for classification of the four road surfaces is possible. The computations are not complex allowing it to perform as a preview sensor for vehicles. There is no significant computational difference between the two- and the three-dimensional system. Using the ratio between the wavelengths gives a reliable method that is not affected by changes in the intensity levels of reflection. The low probability of wrong classification for the surfaces $\left(P_{\text {wrong }}=1 \%\right)$ for the measurements seems promising for measuring surfaces that are not as distinct as in this investigation, for example, water and ice or ice and snow together. Some of the measurements that are classified as wrong surfaces may be caused by measurement errors as not pressing the modified spectrometer against the sphere, so it points to the center of the illumination. As the number of measurements is almost 10,000 , this is a probable cause of errors.

\section{Conclusions}

It is possible to recognize different road conditions with light in the NIR spectrum of light in a laboratory environment. Using only the backscattered light, as is preferable in a preview sensor, it is shown in this investigation and for these measurements that the four road conditions-dry, wet, icy, and snowy asphaltare detectable with a rather low possibility of wrong classification of $1 \%-4 \%$ depending on the number of measurements. These positive results are encouraging for the development of a system to work in actual automotive scenarios.

\section{References}

1. C.-G. Wallman and H. åström, "Friction measurement methods and the correlation between road friction and traffic safety," VTI meddelande 911A (2001).

2. M. Yamada, T. Oshima, K. Ueda, I. Horiba, and S. Yamamoto, "A study of the road surface condition detection technique for deployment on a vehicle," JSAE Rev. 24, 183-188 (2003).

3. F. Holzwarth and U. Eichhorn, "Noncontact sensors for road conditions," Sens. Actuators 37, 121-127 (1993).

4. T. Ogura, I. Kageyama, K. Nasukawa, Y. Miyashita, H. Kitagawa, and Y. Imada, "Study on road surface sensing system for snow and ice road," ISAE Rev. 23, 333-339 (2002).

5. E. Hecht, Optics, 3rd ed. (Addison-Wesley Longman, 1998).

6. M. Herold and D. Roberts, "Spectral characteristics of asphalt road aging and deterioration: implications for remote-sensing applications," Appl. Opt. 44, 4327-4334 (2005).

7. A. W. Nolin and J. Dozier, "A hyperspectral method for remotely sensing the grain size of snow," Remote Sensing Environ. 74, 207-216 (2000)

8. E. A. Cloutis, "Spectral reflectance properties of hydrocarbonsremote-sensing implications," Science 245, 165-168 (1989). 